\title{
Helical Tomotherapy Based Image-Guided Intensity-Modulated Radiation Therapy for Complex, Irregular, Residual, Recurrent, Progressive Benign/ Low-Grade Meningiomas
}

Tejpal Gupta*, Tabassum Wadasadawala, Reena Phurailatpam, Siji Nojin Paul and Rakesh Jalali

Department of Radiation Oncology, Advanced Centre for Treatment Research and Education in Cancer (ACTREC) \& Tata Memorial Hospital (TMH), Tata Memorial Centre, Kharghar, Navi Mumbai, India

\begin{abstract}
Aim: To report mature outcomes of helical Tomotherapy $(\mathrm{HT})$-based image-guided intensity-modulated radiation therapy (IMRT) in benign/low-grade meningiomas.

Methods: Nineteen consecutive patients with 22 complex, irregular, residual, recurrent, or progressive benign/ low-grade meningiomas were treated on HT and followed up clinico-radiologically. Tumor control was defined as lack of evidence of tumor progression on serial imaging. Progression-free survival (PFS) was calculated from date of initiation of HT till imaging-defined progression or sustained neurologic worsening whichever occurred earlier.

Results: The median age of the study cohort was 40 years (range $15-72$ years) and included $13(68 \%)$ females. HT achieved excellent target volume coverage, good high-dose conformality and homogeneity with exquisite sparing of surrounding normal critical structures. Acute toxicity of HT was mild and self-limiting. Using standard response evaluation criteria, 18 of $22(83 \%)$ lesions were stable on first response assessment, while $4(17 \%)$ lesions showed partial response. Pre-existent neuro-deficits present in 13 patients prior to HT either improved or remained stable following irradiation in all but one patient. Only 1 patient $(5 \%)$ developed cataract necessitating extraction. Two $(10.5 \%)$ patients had clinical and/or radiological progression on follow-up. With a median follow-up of 32 months (inter-quartile range $27-45$ months), the 5-year clinico-radiological PFS was $89.2 \%$. All 19 patients were alive for a 5 -year overall survival of $100 \%$.
\end{abstract}

Conclusion: HT-based image-guided IMRT for benign/low-grade meningiomas achieves excellent high-dose conformality with minimal acute and late morbidity resulting in excellent long-term outcomes prompting its use in routine clinical practice.

Keywords: Benign; Low-grade; Meningioma; Peri-orbital; Skullbase; Tomotherapy

\section{Introduction}

Meningiomas are the most common primary central nervous system (CNS) neoplasms [1,2] constituting nearly $25-30 \%$ of all primary brain tumors. Most of these tumors are indolent, slowgrowing and histologically benign (grade I) based on the current World Health Organization (WHO) classification [3], although recent times have witnessed an increasing proportion of patients being diagnosed with atypical or invasive tumors (WHO grade II). Surgical resection remains the primary treatment of choice for the vast majority of meningiomas. In accessible sites, gross total resection for benign meningiomas results in good long-term control and excellent survival [4]. However, meningiomas are frequently located at anatomically difficult to access sites (peri-orbital, sphenoidal, petro-clival); eloquent areas (parietal para-sagittal, posterior fossa); or involve vital neurovascular structures (sinus, nerves) precluding aggressive surgical resection. The use of external beam radiation therapy (RT) has steadily increased over the years for residual incompletely excised tumors (adjuvant setting) as well as an alternative to surgery (definitive setting) for orbital and central skull-base tumors to achieve long-term control [5]. In addition, RT remains an integral part of management of recurrent/progressive meningiomas and histologically higher grade (atypical, invasive, or anaplastic) tumors [6]. Due to their complex, irregular shape and proximity to vital critical structures, meningiomas present a therapeutic challenge to planning and delivery of radiation therapy, and are ideally suited to the newer high-precision techniques such as stereotactic conformal radiation therapy (SCRT) or intensitymodulated radiation therapy (IMRT).

Helical Tomotherapy (HT) has recently emerged as a promising and novel technology [7] for the planning and delivery of highly conformal doses to target volumes across various sites including brain with excellent conformal avoidance of surrounding organs-at-risk (OARs). A 6 MV linear accelerator (linac) mounted on a ring gantry continuously rotates around the patient to deliver radiation in a helical mode as the patient translates through the ring. Herein we report mature outcomes on our previous encouraging experience of HT-based image-guided IMRT with a focus on benign/low-grade meningiomas.

\section{Materials and Methods}

Nineteen patients with meningiomas of varied histological subtypes treated consecutively on HT (HI-ART II version 3.1, Tomotherapy Inc,

*Corresponding author: Dr. Tejpal Gupta, Associate Professor, Radiation Oncology, ACTREC, Tata Memorial Centre, Kharghar, Navi Mumbai:410210, India, Tel: 91-22-27405000; Fax: 91-22-27405061; E-mail: tejpalgupta@rediffmail.com

Received June 11, 2012; Accepted June 21, 2012; Published June 25, 2012

Citation: Gupta T, Wadasadawala T, Phurailatpam R, Paul SN, Jalali R (2012) Helical Tomotherapy Based Image-Guided Intensity-Modulated Radiation Therapy for Complex, Irregular, Residual, Recurrent, Progressive Benign/Low-Grade Meningiomas. J Nucl Med Radiat Ther S3:004. doi:10.4172/2155-9619.S3-004

Copyright: ( 2012 Gupta T, et al. This is an open-access article distributed under the terms of the Creative Commons Attribution License, which permits unrestricted use, distribution, and reproduction in any medium, provided the original author and source are credited. 
Citation: Gupta T, Wadasadawala T, Phurailatpam R, Paul SN, Jalali R (2012) Helical Tomotherapy Based Image-Guided Intensity-Modulated Radiation Therapy for Complex, Irregular, Residual, Recurrent, Progressive Benign/Low-Grade Meningiomas. J Nucl Med Radiat Ther S3:004. doi:10.4172/2155-9619.S3-004

Madison, WI, USA) during the period of January 2008 to February 2010, and followed up clinico-radiologically constitute the study cohort. All patients underwent a pre-RT (baseline) comprehensive neurological examination and other specific assessments according to existent deficits and/or anatomic location viz. ophthalmological, endocrinological, and auditory evaluation for peri-orbital, para-sellar, and petro-clival lesions respectively.

\section{HT planning and evaluation}

The process of planning, delivery, and verification on HT have been described in detail previously [8]. The $6 \mathrm{MV}$ beam in Tomotherapy HI-ART II is collimated and modulated by 64 pairs of pneumatically driven binary multi-leaf collimators (MLCs) having $0.625 \mathrm{~cm}$ projected leaf width at isocenter [7]. It uses an inverse treatment planning process based on iterative least squares minimization of an objective function with the dose being calculated using a superpositionconvolution algorithm [7]. Typical planning parameters used for optimization and dose computation were a fan beam thickness (FBT) of $2.5 \mathrm{~cm}$, pitch of 0.3 and a modulation factor between 2 to 3.5 . For very small targets (such as peri-orbital tumors), an FBT of $1 \mathrm{~cm}$ was used sometimes to achieve better conformality. Directional blocking was employed wherever appropriate to prevent beamlets from entering through critical OARs (eg. eyes). HT plans were evaluated qualitatively and quantitatively using standardized dose-volume indices in terms of target volume coverage, dose homogeneity, dose conformity, and OAR sparing. For reporting purposes, maximum doses were specified as maximum dose $\left(D_{m a x}\right)$ to a minimum yet clinically significant volume (1\%). Similarly minimum doses were specified as the minimum dose $\left(D_{\min }\right)$ received by $99 \%$ of the volume. This eliminates isolated dose peaks and troughs within clinically insignificant volumes (single or few voxels). Target volume coverage and dose homogeneity were assessed as the volume of PTV receiving at least $95 \%\left(\mathrm{~V}_{95 \%}\right)$ and $107 \%\left(\mathrm{~V}_{107 \%}\right)$ of the prescribed dose. Dose homogeneity was evaluated quantitatively using the dose homogeneity index (DHI) defined as a ratio of the difference between dose to $5 \%$ volume $\left(D_{5 \%}\right) \& 95 \%$ volume $\left(D_{95 \%}\right)$ by the mean dose $\left(\mathrm{D}_{\text {mean }}\right)$ to the PTV expressed as a percentage (DHI $\left.=\left\{D_{5 \%}-D_{95 \%}\right\} / D_{\text {mean }} X 100 \%\right)$. The conformation of therapeutic dose volume to the target volume was estimated using the conformity index (CI) as defined by Paddick [8] [CI $\left.=\left\{\mathrm{V}_{\mathrm{T}, \mathrm{Pi} \times} \mathrm{V}_{\mathrm{T}, \mathrm{Pi}}\right\} /\left\{\mathrm{V}_{\mathrm{T}} \times \mathrm{V}_{\mathrm{Pi}}\right\}\right]$, where $\mathrm{V}_{\mathrm{T}, \mathrm{Pi}}$ is the volume of target enclosed by the prescription dose; $\mathrm{V}_{\mathrm{Pi}}$ is the volume of tissues including target covered by the prescription dose; and $\mathrm{V}_{\mathrm{T}}$ is the volume of target). Maximum and mean dose $\left(\mathrm{D}_{\max }\right.$ and $\mathrm{D}_{\text {mean }}$ ) in Gy was recorded for estimation of OAR sparing.

All patients underwent response assessment imaging at first followup (2-3 months after completion of radiotherapy). Subsequent followups were scheduled at 3-4 monthly intervals for the first 2 years and 6-monthly intervals thereafter till 5 years, with imaging done annually or earlier in case of neurologic worsening. Progression-free survival (PFS) was calculated from date of initiation of HT-based IMRT till magnetic resonance imaging (MRI)-defined progression or sustained neurologic worsening whichever occurred earlier. Temporary reversible worsening ascribable to seizures, infection, or other unrelated causes was not considered an event for PFS. Overall survival was calculated from date of initiation of HT-based IMRT till death or last follow-up. All time-to-event analysis was done using the product-limit method of Kaplan-Meier and expressed as 5-year estimates with 95\% confidence intervals $(95 \% \mathrm{CI})$. The close-out date for survival analysis was February 2012. All analyses were done on SPSS version 17.0.

\section{Results}

During the specified period, 45 patients with benign/low-grade
CNS tumors were accrued and treated on a prospective generic protocol of HT-based image-guided IMRT after written informed consent. Amongst these, 19 patients with 22 meningiomas constitute the present study cohort. The median age at the time of irradiation on HT was 40 years (range 15-72 years) and females $(n=13,68 \%)$ were predominant consistent with a typical meningioma cohort. Baseline (pre-HT) tumor characteristics are described in Table 1and Figure 1 gives an insight into the spectrum of meningiomas (site, size, shape) that were treated on the study. Three patients had multiple lesions. One patient had a high parietal lesion (excised completely) and an imaging-defined right optic nerve sheath meningioma; another patient with previously treated stable petro-clival meningioma had cervical spinal (decompressed) and imaging-defined sacral deposit; while the third patient, a known case of neurofibromatosis-2 (NF2) had bilateral acoustic schwannoma, optic nerve sheath meningioma, and an enplaque meningioma growing along the cerebellar convexity. The main indications for RT were post-operative adjuvant for completely resected tumors $(n=1)$; gross residual disease after subtotal resection/

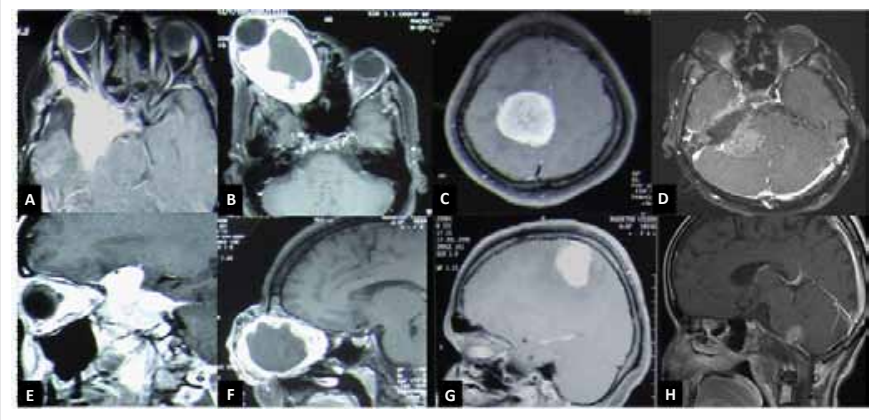

Figure 1: Axial (upper panel, A-D) and sagittal (lower panel, E-H) postcontrast MRI scans showing the spectrum of meningiomas treated on helical tomotherapy.

\begin{tabular}{|l|l|}
\hline Tumor characteristics & Number of patients (\%) \\
\hline Presentation & \\
At first presentation/initial diagnosis & $09(47.4 \%)$ \\
Recurrent/progressive tumor (after prior therapy) & $10(52.6 \%)$ \\
\hline Laterality & \\
Right & $14(63.7 \%)$ \\
Left & $05(22.7 \%)$ \\
Midline (including 2 spinal lesions) & $03(13.6 \%)$ \\
\hline Location & \\
Skull-base & $04(18.2 \%)$ \\
Peri-orbital & $05(22.8 \%)$ \\
Parietal & $06(27.3 \%)$ \\
Frontal & $02(09.1 \%)$ \\
Temporal & $01(04.5 \%)$ \\
Occipital & $01(04.5 \%)$ \\
Cerebellar & $01(04.5 \%)$ \\
Spinal & $02(09.1 \%)$ \\
\hline Number of lesions & \\
Single lesion & $16(84.2 \%)$ \\
Multiple (3 patients with 2 discrete lesions each) & $03(15.8 \%)$ \\
\hline Histological subtype (grade) & \\
Meningothelial (grade I) & $07(33.3 \%)$ \\
Angiomatous (grade I) & $01(04.8 \%)$ \\
Psammomatous (grade I) & $01(04.8 \%)$ \\
Transitional (grade I) & $01(04.8 \%)$ \\
Fibroblastic (grade I) & $01(04.8 \%)$ \\
Syncitial (grade I) & $01(04.8 \%)$ \\
Chordoid (grade II) & $03(14.2 \%)$ \\
Atypical (grade II) & $02(09.5 \%)$ \\
Unverified (including patients with multiple tumors) & $05(19.0 \%)$ \\
\hline
\end{tabular}

Table 1: Baseline tumor characteristics of the study cohort ( $\mathrm{N}=19$ patients, 22 lesions). 
Citation: Gupta T, Wadasadawala T, Phurailatpam R, Paul SN, Jalali R (2012) Helical Tomotherapy Based Image-Guided Intensity-Modulated Radiation Therapy for Complex, Irregular, Residual, Recurrent, Progressive Benign/Low-Grade Meningiomas. J Nucl Med Radiat Ther S3:004. doi:10.4172/2155-9619.S3-004

biopsy $(n=6)$; recurrent/progressive tumor after failed prior therapy $(\mathrm{n}=10)$, and definitive primary RT for symptomatic tumor $(\mathrm{n}=2)$. All 3 patients with multiple lesions received RT to the index lesion as well as to the incidentally detected imaging-defined meningiomas as definitive primary RT for asymptomatic tumor $(\mathrm{n}=3)$. Gross tumor was treated to a higher dose using simultaneous integrated boost in 3 of 5 patients with peri-orbital meningiomas. HT plans were evaluated qualitatively and quantitatively using standardized dose-volume indices. Tables 2 and 3 show summary dosimetric parameters for the planning target volume (PTV) and OARs for the entire study cohort. Despite the complex and irregularly shaped target volumes, HT was able to achieve excellent PTV coverage, good high-dose conformality and homogeneity, with exquisite OAR sparing (Figure 2).

\section{Toxicity outcomes}

All patients were reviewed at least once weekly during the course of RT. The acute toxicity of HT-based image-guided IMRT was mild and self-limiting, with no patients developing any severe (grades III-IV) acute toxicity. All patients had mild hyperpigmentation and occasional dry desquamation (grade I-II dermatitis). Patients with per-orbital tumors had mild (grade I-II) ocular toxicity in the form of conjunctival congestion, itching, and occasional watering that responded to topical steroids. Pre-HT formal ophthalmological examination revealed diminished visual acuity in $7(37 \%)$ patients with complete loss of vision in $2(10.5 \%)$ patients in the ipsilateral eye. Vision in the contraletral eye was unaffected in all tested patients at baseline. Following radiotherapy, visual function improved in $5(71.5 \%)$ patients and remained stable in $1(14 \%)$ patient for a visual preservation rate of $85.5 \%$. One patient had progressive worsening of visual function due to long-standing disease. Only 1 (5\%) patient developed cataract in the ipsilateral eye necessitating extraction (grade III late toxicity). Visual function in the contralateral eye remained unaffected in all patients on follow-up. Cranial nerve palsies present in 6 patients at baseline either improved $(n=3)$ or stabilized $(n=3)$. Similarly, motor deficits noted in

\begin{tabular}{|l|l|}
\hline Median PTV volume (range) & $87.8 \mathrm{cc}(14.8-380 \mathrm{cc})$ \\
\hline Median prescription dose (range) & $54 \mathrm{~Gy}(50-60 \mathrm{~Gy})$ \\
\hline Median number of fractions (range) & 30 fractions $(28-30)$ \\
\hline Mean $\mathrm{V}_{95 \%}$ (standard deviation) & $99.8 \%(0.70)$ \\
\hline Mean $\mathrm{V}_{107 \%}$ (standard deviation) & $0.0 \%(0.42)$ \\
\hline Dose Homogeneity Index (standard deviation) & $0.07(0.07)$ \\
\hline Conformity Index (standard deviation) & $0.75(0.07)$ \\
\hline
\end{tabular}

Table 2: Dosimetric parameters of planning target volume (PTV) for the study cohort.

\begin{tabular}{|l|l|l|}
\hline OARs & $\begin{array}{l}\text { Mean of mean dose } \\
\text { (standard deviation) }\end{array}$ & $\begin{array}{l}\text { Mean of maximum } \\
\text { dose }\end{array}$ \\
\hline Brain stem & $19.6 \mathrm{~Gy}(14.6)$ & $36.7 \mathrm{~Gy}$ \\
\hline Optic chiasm & $31.9 \mathrm{~Gy}(20.1)$ & $36.5 \mathrm{~Gy}$ \\
\hline Ipsilateral optic nerve & $18.4 \mathrm{~Gy}(18.1)$ & $27.4 \mathrm{~Gy}$ \\
\hline Contralateral optic nerve & $10.9 \mathrm{~Gy}(7.8)$ & $17.1 \mathrm{~Gy}$ \\
\hline Ipsilateral lens & $7.0 \mathrm{~Gy}(9.0)$ & $8.9 \mathrm{~Gy}$ \\
\hline Contralateral lens & $3.8 \mathrm{~Gy}(2.4)$ & $8.8 \mathrm{~Gy}$ \\
\hline Ipsilateral eye & $13.8 \mathrm{~Gy}(14.8)$ & $23.7 \mathrm{~Gy}$ \\
\hline Contralateral eye & $3.8 \mathrm{~Gy}(2.4)$ & $7.4 \mathrm{~Gy}$ \\
\hline Ipsilateral temporal lobe & $30.8 \mathrm{~Gy}(18.9)$ & $45.7 \mathrm{~Gy}$ \\
\hline $\begin{array}{l}\text { Contralateral temporal } \\
\text { lobe }\end{array}$ & $14.3 \mathrm{~Gy}(8.3)$ & $28.7 \mathrm{~Gy}$ \\
\hline Pituitary & $31.1 \mathrm{~Gy}(21.8)$ & $34.1 \mathrm{~Gy}$ \\
\hline Whole brain & $19.1 \mathrm{~Gy}(20.4)$ & $48.1 \mathrm{~Gy}$ \\
\hline
\end{tabular}

Table 3: Dosimetric parameters of organs-at-risk (OARs) for the study cohort.

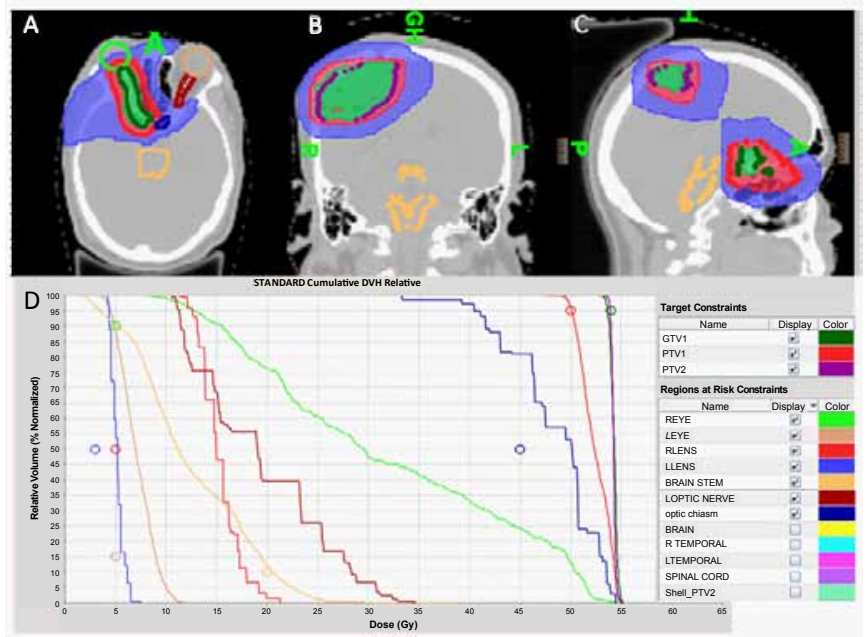

Figure 2: Tomoplan of a patient with two separate lesions treated simultaneously in one single plan. Dose wash of 54Gy (green), 50Gy (red), and $30 \mathrm{~Gy}$ (blue) is displayed showing excellent target volume coverage of right optic nerve sheath meningioma in axial section $(A)$, high parietal meningioma in coronal section (B), and both lesions in sagittal section (C). Corresponding dose-volume histogram of the same patient is also shown.

$4(21 \%)$ patients at baseline either improved $(n=3)$ or remained stable $(\mathrm{n}=1)$ on follow-up.

\section{Clinico-radiological outcomes}

Tumor control was defined as lack of evidence of tumor progression on serial imaging. Using standard response evaluation criteria [9], 18 of 22 (83\%) lesions were stable on first response assessment, while 4 (17\%) lesions showed partial response. Two (10.5\%) patients had clinical and/ or radiological progression on follow-up. One of them, with a previously treated and stable petro-clival meningioma had presented with discrete cervical spinal and sacral lesions. The cervical spine was decompressed surgically and confirmed to be atypical meningioma histologically. Subsequently, she received RT to the cervical and sacral spine on HT. Seven months later, an MRI done for modest clinical deterioration showed increase in the cervical spinal lesion, but stable sacral lesion. In addition, new lesions with patchy enhancement were also noted in the cerebellum and medulla, which could represent new deposits or posttreatment changes. Her symptoms improved with steroids and she continues to have stable deficits on further follow-up. The other patient with a para-sagittal meningioma had recurred 8 years after initial treatment (surgery followed by adjuvant radiotherapy). Following sub-total re-excision, he underwent re-irradiation of the tumor bed on HT and was clinically/neurologically controlled. He was detected to have imaging-defined progression on surveillance scan 21 months after re-irradiation; subsequently patient worsened neurologically also, but is presently alive with stable deficits on follow-up. With a median follow up of 32 months (inter-quartile range 27-45 months), the 5-year Kaplan-Meir estimate (95\%CI) of clinico-radiological PFS (Figure 3) for the study cohort was $89.2 \%(82-96.4 \%)$. All patients are alive at the time of this analysis for a 5 -year overall survival of $100 \%$.

\section{Discussion}

Meningiomashave traditionallybeen considered the neurosurgeon's domain and surgical excision of the tumor with its dural base remains the most common primary or initial management. Simpson et al. [10] proposed a grading system based on extent of surgical resection with 
Citation: Gupta T, Wadasadawala T, Phurailatpam R, Paul SN, Jalali R (2012) Helical Tomotherapy Based Image-Guided Intensity-Modulated Radiation Therapy for Complex, Irregular, Residual, Recurrent, Progressive Benign/Low-Grade Meningiomas. J Nucl Med Radiat Ther S3:004. doi:10.4172/2155-9619.S3-004

Page 4 of 6

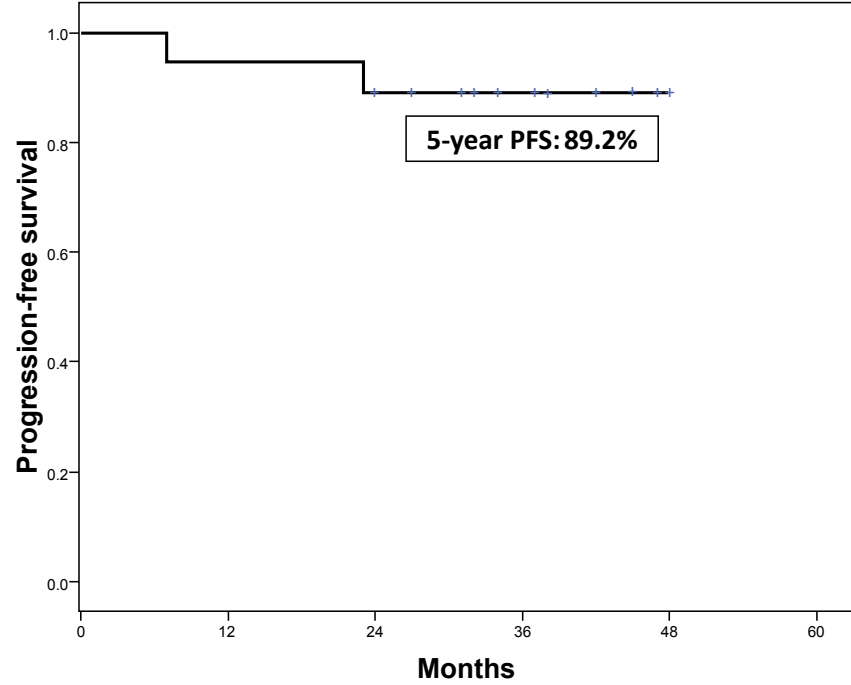

Figure 3: Kaplan-Meir estimate of clinico-radiological progression-free survival of study cohort treated on helical tomotherapy with image-guided intensity modulated radiation therapy.

5 -year local recurrence rates of $9 \%, 19 \%$, and $29 \%$ after Simpson grades 1-3 excision respectively for benign meningiomas. Meningiomas were perceived to be relatively 'radio-resistant' and the anticipated morbidity with conventional RT prevented its use for inoperable or sub-totally resected meningiomas [11,12]. However, with the understanding that meningiomas typically remain stable or regress slowly following RT, several investigators have now reported consistent benefit in outcomes with radiotherapy. A recent analysis of over 3500 patients of WHO grade I meningioma treated with either gross total resection or subtotal resection with or without adjuvant $\mathrm{RT}$, reported an improvement in 10-year PFS from $50-90 \%$ with the addition of RT [13].

Presently, RT is an integral component in the management of residual, recurrent, and/or progressive benign/low-grade meningiomas. However, close proximity to several critical structures mandates careful selection of treatment technique to achieve durable local control with minimum long-term morbidity. Dosimetric comparisons of several high-precision irradiation techniques using a variety of planning and delivery platforms have been attempted to define the most optimal technique for benign intracranial tumors including meningiomas. It is widely accepted that SCRT with uniform-intensity beams is able to maximally restrict doses to non-target tissues for small and spherical targets in the brain [14]. Larger, irregular, or complex-shaped targets may necessitate the use of multiple isocentres in SCRT, resulting in increased in homogeneity and decreased conformality. Such tumors may benefit the most from non-uniform intensity beams i.e. IMRT, with or without stereotactic-guidance. We have previously shown that non-coplanar stereotactic IMRT is dosimetrically superior to SCRT in a range of benign brain tumors [15]. However, in another study from Netherlands dynamic conformal arc-based SCRT was superior to stereotactic IMRT [16]. Yartsev et al.[17] reported better target dose uniformity with comparable OAR sparing in the first dosimetric comparison of HT with other advanced photon irradiation techniques in patients with small brain tumors. Subsequently, one of the coauthors on the study, compared the potential benefits and limitations of arcbased SCRT, linac-based IMRT, HT, CyberKnife (CK), and intensitymodulated multiple dynamic arc therapy (AMOA) in the same dataset
[18]. HT, AMOA, and linac-based IMRT were superior to CK and SCRT for target volume coverage. All techniques respected planning objectives for OARs, with a tendency of CK and SCRT to better spare brain stem and normal uninvolved brain. HT, however, provided the best overall combination of indices. In a more recent companion publication [19], the same HT plans were compared with another novel platform, RapidArc (Varian Medical Systems, USA), a planning and delivery approach based on volumetric intensity-modulated arc therapy allowing optimization of single coplanar arcs with concomitant dynamic optimization of MLC shapes, dose rate, and gantry speed. All techniques (HT, RapidArc, linac-based IMRT) resulted in equivalent PTV and OAR sparing, with RapidArc plans done using 120-leaf highdefinition MLCs providing the best combination.

Linac-based stereotactic IMRT, arc-based SCRT, or fixed-field SCRT has been compared to HT for skull-base tumors [20]. HT plans compared to non-coplanar linac-based stereotactic IMRT plans showed increased prescription isodose to target volume ratios, variable change in homogeneity index, similar equivalent uniform dose values, and comparable mean normalized total dose for OARs. HT resulted in an increase in low-dose isodose volumes. HT provided superior in homogeneity index compared to coplanar stereotactic IMRT plans. There was a distinct advantage in using non-coplanar beam arrangements for skull-base tumors. However, in case of inferior spread of disease, limiting the ability to use non-coplanar beam arrangement, HT could provide a comparable plan, with potentially superior homogeneity. In a more recent attempt to achieve dose escalation, Estall et al.[21], compared linac-based coplanar IMRT with HT in 5 patients with skull-base meningioma for plan efficacy and efficiency. Both techniques resulted in similar plan efficacy achieving safe dose escalation (to $60 \mathrm{~Gy}$ ) with highly conformal PTV coverage as well as OAR sparing to below specified absolute tolerance levels. However, the average beam-on time for a single fraction was 6.7 minutes for HT as compared to 18.4 minutes for linac-based IMRT, suggesting significant resource-implications in busy departments. In a companion publication [22], the authors also proposed practical IMRT planning class solutions for skull-base tumors (seven field coplanar beam arrangement for central tumors and a five-field non-coplanar or coplanar beam arrangement for lateralized lesions, with smallest available MLC leaf width) for safe dose escalation to $60 \mathrm{~Gy}$.

There is a distinct advantage in using non-coplanar beam arrangements for skull-base tumors, by avoiding entry and exit of beams through OARs, which may be considered an inherent limitation of HT (exclusive coplanar nature of treatment delivery). However, we have been able to overcome that to a great extent by utilizing directional block (preventing beamlet entry) and sometimes even complete blocking (avoiding entry as well as exit) of the OAR during optimization. We would like to emphasize that plan comparison of radiotherapy techniques and modalities can be challenging and must be interpreted with caution due to differences in prescription, normalization, optimization and calculation algorithms, voxel and grid-size, and overlap priority, particularly if the differences are small.

The safety and efficacy of IMRT in benign/low-grade meningiomas has now been firmly established by several prospective clinical outcome studies (Table 4) [23-28]. Most series report excellent disease outcomes at 3-5 years with Kaplan-Meier estimates of local control and/or PFS well over $90 \%$ and acceptable significant late toxicity (around 5\%). Encouraging early clinical data of HT-based image-guided IMRT for meningiomas has also been reported recently. Based on their prior experience with IMRT, researchers at the University of Heidelberg 
Citation: Gupta T, Wadasadawala T, Phurailatpam R, Paul SN, Jalali R (2012) Helical Tomotherapy Based Image-Guided Intensity-Modulated Radiation Therapy for Complex, Irregular, Residual, Recurrent, Progressive Benign/Low-Grade Meningiomas. J Nucl Med Radiat Ther S3:004. doi:10.4172/2155-9619.S3-004

Page 5 of 6

\begin{tabular}{|c|c|c|c|c|c|c|c|c|}
\hline Author (ref) & No. of pts & IMRT platform & Median volume & Median dose & $\begin{array}{l}\text { Median follow- } \\
\text { up }\end{array}$ & PFS/local control & $\begin{array}{l}\text { Overall } \\
\text { survival }\end{array}$ & Late toxicity \\
\hline Uy (23) & 40 & Peacock-NOMOS & $20.2 \mathrm{cc}$ & 50.4 Gy & 30 months & 93\% (5-year) & 89\% (5-year) & $5 \%$ \\
\hline Pirzkall (24) & 20 & $\begin{array}{l}\text { KonRad, Siemens } \\
\text { CORVUS-NOMOS }\end{array}$ & $108 \mathrm{cc}$ & 57 Gy & 36 months & $\begin{array}{l}\text { 100\% (imaging) } \\
95 \% \text { (clinical) }\end{array}$ & NR & 0 \\
\hline Sajja (25) & 35 & Peacock-NOMOS & NR & 50.4 Gy & 19.1 months & 97\% (3-year) & NR & 0 \\
\hline $\begin{array}{l}\text { Milker-Zabel } \\
(26)\end{array}$ & 94 & KonRad, Siemens & $81.4 \mathrm{cc}$ & $57.6 \mathrm{~Gy}$ & 52 months & 95\% (5-year) & 97\% (5-year) & $4 \%$ \\
\hline Combs (27) & 12 & $\begin{array}{l}\text { Helical } \\
\text { Tomotherapy }\end{array}$ & NR & 58 Gy & 12 months & $\begin{array}{l}\text { 4/12 pts (all grade II-III) } \\
\text { progressed }\end{array}$ & $100 \%$ & $8 \%$ \\
\hline Luis (28) & 28 & $\begin{array}{l}\text { Helical } \\
\text { Tomotherapy }\end{array}$ & $43.3 \mathrm{cc}$ & $51.7 \mathrm{~Gy}$ & 7.5 months & $100 \%$ (imaging) & $100 \%$ & NR \\
\hline Present study & 19 & $\begin{array}{l}\text { Helical } \\
\text { Tomotherapy }\end{array}$ & $87.8 \mathrm{cc}$ & $54 \mathrm{~Gy}$ & 32 months & $89.2 \%$ (5-year) & $100 \%$ (5-year) & $5 \%$ \\
\hline
\end{tabular}

Pts=patients; PFS=progression-free survival; NR=not reported

Table 4: Prospective clinical studies of intensity-modulated radiation therapy (IMRT) in meningiomas.

adopted the HT platform [27] for treating 12 patients with multiple or complex meningiomas (skull-base and para-spinal). Eight patients were treated with primary radiotherapy, while 4 patients were re-irradiated for recurrent/progressive lesions. Treatment was well tolerated, with no severe acute or significant late toxicity. All patients were alive at the time of reporting, although 4 of 12 patients ( 2 of 5 with atypical and 2 of 2 with anaplastic histology) developed progression at 2, 4, 17 , and 29 months after radiotherapy, reflecting the poor biology of higher grade meningiomas. Schiappacasse et al. [28] recently reported their preliminary experience of treating 28 consecutive patients with complex-shaped meningiomas close to the optic pathway on HT as primary (36\%), adjuvant (32\%), or rescue (32\%) treatment after postsurgical progression. Acute toxicity was mild (grade I) and transitory that included headache (36\%), ocular pain/dryness $(29 \%)$, dermatitis (25\%), and somnolence (20\%). All patients had radiological stabilization of disease and no significant late sequelae were documented at a maximal follow-up of 3 years.

Although the number of patients included in our study was relatively small $(n=19)$, its strength lies in the demonstration of excellent intermediate to long-term efficacy (5-year PFS of around 90\%) and safety ( $5 \%$ severe late toxicity) of HT-based IMRT in meningiomas at a relatively mature follow-up (median of 32 months) as opposed to comparatively shorter follow-up times reported by the others $[27,28]$. A critical appraisal of contemporary advanced photon irradiation techniques coupled with encouraging clinical outcome data favours the adoption of HT for large, irregularly-shaped, and complex benign brain tumors (such as meningiomas) in routine clinical practice.

\section{Conclusion}

Radiotherapy remains an integral component in the management of residual, recurrent, and/or progressive benign/low-grade meningiomas. Given their complex, irregular shape and proximity to several critical structures, careful selection of treatment technique is warranted for maximizing local control and minimising morbidity. Image-guided IMRT on HT achieves excellent high-dose conformality and OAR sparing with minimal acute and late morbidity and results in excellent long-term outcomes prompting its use in routine clinical practice.

\section{Acknowledgements}

All neurosurgeons for referring the patients for radiotherapy and all patients for having consented for treatment with helical Tomotherapy.

Patients' consent for publication: The clinical material submitted herewith is completely anonymous and patients have provided consent for publication.
Financial support: Patients were treated on an Institutional Review Board approved protocol wherein radiation therapy charges were waived off by the institute.

\section{References}

1. Claus EB, Bondy ML, Schildkraut JM, Wiemels JL, Wrensch M, et al. (2005) Epidemiology of intracranial meningioma. Neurosurgery 57: 1088-1095.

2. CBTRUS (2012) Statistical Report: Primary Brain and Central Nervous System Tumors Diagnosed in the United States in 2004-2008. Central Brain Tumor Registry of the United States, Hinsdale, IL, USA

3. Louis DN, Ohgaki H, Wiestler OD, Cavenee WK, Burger PC, et al. (2007) The $2007 \mathrm{WHO}$ classification of tumours of the central nervous system. Acta Neuropathol 114: 97-109.

4. Stafford SL, Perry A, Suman VJ, Meyer FB, Scheithauer BW, et al. (1998) Primarily resected meningiomas: outcome and prognostic factors in 581 Mayo Clinic patients, 1978 through 1988. Mayo Clin Proc 73: 936-942.

5. Nutting C, Brada M, Brazil L, Sibtain A, Saran F, et al. (1999) Radiotherapy in the treatment of benign meningioma of the skull base. J Neurosurg 90: 823827

6. Pourel N, Auque J, Bracard S, Hoffstetter S, Luporsi E, et al. (2001) Efficacy of external fractionated radiation therapy in the treatment of meningiomas: a 20 year experience. Radiother Oncol 61: 65-70.

7. Mackie TR, Holmes T, Swerdloff S, Reckwerdt P, Deasy JO, et al. (1993) Tomotherapy: a new concept in the delivery of dynamic conformal radiotherapy. Med Phys 20: 1709-1719.

8. Gupta T, Wadasadawala T, Master Z, Phurailatpam R, Pai-Shetty R, et al (2011) Encouraging early clinical outcomes with helical tomotherapy-based image-guided intensity-modulated radiation therapy for residual, recurrent, and/or progressive benign/low-grade intracranial tumors: a comprehensive evaluation. Int J Radiat Oncol Biol Phys 82: 756-764.

9. Miller AB, Hoogstraten B, Staquet M, Winkler A (1981) Reporting results of cancer treatment. Cancer 47: 207-214.

10. Simpson D (1957) The recurrence of intracranial meningiomas after surgical treatment. J Neurol Neurosurg Psychiatry 20: 22-39.

11. Miralbell R, Linggood RM, de la Monte S, Convery K, Munzenrider JE, et al. (1992) The role of radiotherapy in the treatment of subtotally resected benign meningiomas. J Neuro-Oncol 13: 157-164

12. Jung HW, Yoo H, Paek SH, Choi KS (2000) Long-term outcome and growth rate of subtotally resected petro-clival meningiomas: experience with 38 cases. Neurosurgery 46: 567-574.

13. Gondi V, Tome WA, Mehta MP (2010) Fractionated radiotherapy for intracrania meningiomas. J Neuro-Oncol 99: 349-356.

14. Baumert BG, Norton IA, Davis JB (2003) Intensity-modulated stereotactic radiotherapy vs. stereotactic conformal radiotherapy for the treatment of meningioma located predominantly in the skull base. Int J Radiat Oncol Biol Phys 57: 580-592.

15. Sharma SD, Jalali R, Phurailatpam RD, Gupta T (2009) Does intensity- 
Citation: Gupta T, Wadasadawala T, Phurailatpam R, Paul SN, Jalali R (2012) Helical Tomotherapy Based Image-Guided Intensity-Modulated Radiation Therapy for Complex, Irregular, Residual, Recurrent, Progressive Benign/Low-Grade Meningiomas. J Nucl Med Radiat Ther S3:004. doi:10.4172/2155-9619.S3-004

modulated stereotactic radiotherapy achieve superior target conformity than conventional stereotactic radiotherapy in different intracranial tumours? Clin Oncol 21: 408-416.

16. Wiggenraad RG, Petoukhova AL, Versluis L, van Santvoort JP (2009) Stereotactic radiotherapy of intracranial tumors: a comparison of intensitymodulated radiotherapy and dynamic conformal arc. Int J Radiat Oncol Biol Phys 74: 1018-1026.

17. Yartsev S, Kron T, Cozzi L, Fogliata A, Bauman G (2005) Tomotherapy planning of small brain tumours. Radiother Oncol 74: 49-52.

18. Cozzi L, Clivio A, Bauman G, Cora S, Nicolini G, Pellegrini R, et al (2006) Comparison of advanced irradiation techniques with photons for benign intracranial tumors. Radiother Oncol 80: 268-273.

19. Fogliata A, Clivio A, Nicolini G, Vanetti E, Cozzi L (2009) Intensity modulation with photons for benign intracranial tumours: A planning comparison of volumetric single arc, helical arc and fixed gantry techniques. Radiother Oncol 89: $254-262$

20. Soisson ET, Tome WA, Richards GM, Mehta MP (2006) Comparison of linacbased fractionated stereotactic radiotherapy and tomotherapy treatment plans for skull-base tumors. Radiother Oncol 78: 313-321.

21. Estall V, Fairfoul J, Jena R, Jefferies SJ, Burton KE, et al. (2010) Skull base meningioma - comparison of intensity-modulated radiotherapy planning techniques using the moduleaf micro-multileaf collimator and helical tomotherapy. Clin Oncol 22: 179-184.
22. Estall VJ, Eaton D, Burton KE, Jefferies SJ, Jena R, et al. (2010) Intensitymodulated radiotherapy plan optimization for skull base lesions: practical class solutions for dose escalation. Clin Oncol (R Coll Radiol) 22: 313-320.

23. Uy NW, Woo SY, Teh BS, Mai WY, Carpenter LS, et al. (2002) Intensitymodulated radiation therapy (IMRT) for meningioma. Int $\mathrm{J}$ Radiat Oncol Biol Phys 53: 1265-1270

24. Pirzkall A, Debus J, Haering P, Rhein B, Grosser KH, et al. (2003) Intensity modulated radiotherapy (IMRT) for recurrent, residual, or untreated skull-base meningiomas: preliminary clinical experience. Int J Radiat Oncol Biol Phys 55: 362-372.

25. Sajja R, Barnett GH, Lee SY, Harnisch G, Stevens GH, et al. (2005) Intensitymodulated radiation therapy (IMRT) for newly diagnosed and recurrent intracranial meningiomas: preliminary results. Technol Cancer Res Treat 4: 675-682.

26. Milker-Zabel S, Zabel-du Bois A, Huber P, Schlegel W, Debus J (2007) Intensity-modulated radiotherapy for complex-shaped meningioma of the skul base: long-term experience of a single institution. Int J Radiat Oncol Biol Phys 68: 858-863

27. Combs SE, Sterzing F, Uhl M, Habl G, Schubert K, et al. (2011) Helical tomotherapy for meningiomas of the skull base and in paraspinal regions with complex anatomy and/or multiple lesions. Tumori 97: 484-491.

28. Schiappacasse L, Cendales R, Sallabanda K, Schnitman F, Samblas J (2011) Preliminary results of helical tomotherapy in patients with complex-shaped meningiomas close to the optic pathway. Med Dosim 36: 416-422.
This article was originally published in a special issue, Image-guided radiation therapy handled by Editor(s). Dr. Yunfeng Cui, Thomas Jefferson University Hospital, USA; Dr. James Robert Wong, Morristown Memorial Hospital, USA 